\title{
Weak forms of continuity in I-double gradation fuzzy topological spaces
}

\author{
A Ghareeb ${ }^{1,2}$
}

\begin{abstract}
In this paper, we introduce and characterize double fuzzy weakly preopen and double fuzzy weakly preclosed functions between I-double gradation fuzzy topological spaces and also study these functions in relation to some other types of already known functions.
\end{abstract}

\section{Introduction}

In the history of science, new theories have always been necessary in order for existing scientific theories to progress and this will continue to be true in the future. Two examples of essentially different mathematical theories that deal with the concept of uncertainty are probability theory and the theory of fuzzy sets. Whereas probability theory has a history of around 360 years, the theory of fuzzy sets is little more than 50 years old. Since the 1960s fuzzy methods have entered the scientific and technological world, good theoretical progress (e.g., fuzzy logic, fuzzy probability theory, fuzzy topology, fuzzy algebra) has been made, and there have been technical advances in various areas (e.g., fuzzy control, fuzzy expert systems, fuzzy clustering and data mining).

Chang (1968); Lowen (1976); Šostak (1985); Kubiak (1985); Samanta and Mondal () and many others contributed a lot to the field of Fuzzy Topology. In recent years Fuzzy Topology has been found to be very useful in solving many practical problems. Shihong Du et. al. (2005) are currently working to fuzzify the 9-intersection Egenhofer model Egenhofer and Franzosa (1991); Herring and Egenhofer (1991) for describing topological relations in Geographic Information Systems (GIS) query. In El-Naschie (1998, 2000), El-Naschie has shown that the notion of Fuzzy Topology is applicable to quantum particle physics and quantum gravity in connection with String Theory and $e^{\infty}$ Theory. Tang (2004) has used a slightly changed version of Chang's fuzzy topological space to

\footnotetext{
Correspondence: nasserfuzt@aim.com

${ }^{1}$ Mathematics Department, Faculty of Science, South Valley University, Qena, Egypt

${ }^{2}$ Current address: Department of Mathematics, College of Science in Al-Zulfi, Majmaah University, Al-Zulfi, Kingdom of Saudi Arabia
}

model spatial objects for GIS databases and Structured Query Language (SQL) for GIS.

In this paper, we will introduce the concepts of double fuzzy weakly preopen and double weakly preclosed functions in $I$-double gradation fuzzy topological spaces. Their properties and the relationships between these functions and other functions introduced previously are investigated.

\section{Preliminaries}

Throughout this paper, let $X$ be a nonempty set and $I$ is the closed unit interval $[0,1] . I_{\circ}=(0,1]$ and $I_{1}=[0,1)$. The family of all fuzzy subsets on $X$ denoted by $I^{X}$. By $\underline{0}$ and $\underline{1}$, we denote the smallest and the greatest fuzzy subsets on $X$. For a fuzzy subset $\lambda \in I^{X}, \underline{1}-\lambda$ denotes its complement. Given a function $\tilde{f}: X \rightarrow Y, f(\lambda)$ and $f^{-1}(\lambda)$ define the direct image and the inverse image of $f$, defined by $f(\lambda)(y)=\bigvee_{f(x)=y} \lambda(x)$ and $f^{-1}(v)(x)=v(f(x))$, for each $\lambda \in I^{X}, v \in I^{Y}$, and $x \in X$, respectively. For fuzzy subsets $\lambda$ and $\mu$ in $X$, we write $\lambda q \mu$ to mean that $\lambda$ is quasi coincident (q-coincident) with $\mu$, that is, there exists at least one point $x \in X$ such that $\lambda(x)+\mu(x)>1$. Negation of such a statement is denoted as $\lambda \bar{q} \mu$. Notions and notations not described in this paper are standard and usual.

Definition 2.1. [(Samanta and Mondal (1997, 2002); Garcia and Rodabaugh (2005)] An I-double gradation fuzzy topology $\left(\tau, \tau^{*}\right)$ on $X$ is a pair of maps $\tau, \tau^{*}: I^{X} \rightarrow I$, which satisfies the following properties:

$$
\begin{array}{ll}
\text { (O1) } & \tau(\lambda) \leq 1-\tau^{*}(\lambda) \text { for each } \lambda \in I^{X} . \\
\text { (O2) } & \tau\left(\lambda_{1} \wedge \lambda_{2}\right) \geq \tau\left(\lambda_{1}\right) \wedge \tau\left(\lambda_{2}\right) \text { and } \\
& \tau^{*}\left(\lambda_{1} \wedge \lambda_{2}\right) \leq \tau^{*}\left(\lambda_{1}\right) \vee \tau^{*}\left(\lambda_{2}\right) \text { for each } \lambda_{1}, \lambda_{2} \in I^{X} .
\end{array}
$$

\section{量 Springer}

(C) 2012 Ghareeb; licensee Springer. This is an Open Access article distributed under the terms of the Creative Commons Attribution License (http://creativecommons.org/licenses/by/2.0), which permits unrestricted use, distribution, and reproduction in any medium, provided the original work is properly cited. 
(O3) $\tau\left(\bigvee_{i \in \Gamma} \lambda_{i}\right) \geq \bigwedge_{i \in \Gamma} \tau\left(\lambda_{i}\right)$ and $\tau^{*}\left(\bigvee_{i \in \Gamma} \lambda_{i}\right) \leq \bigvee_{i \in \Gamma} \tau^{*}\left(\lambda_{i}\right)$ for each $\lambda_{i} \in I^{X}$, $i \in \Gamma$.

The triplet $\left(X, \tau, \tau^{*}\right)$ is called an $I$-double gradation fuzzy topological spaces ( $I$-dfts, for short). A fuzzy set $\lambda$ is called an $(r, s)$-fuzzy open $((r, s)$-fo, for short) if $\tau(\lambda) \geq r$ and $\tau^{*}(\lambda) \leq s$. A fuzzy set $\lambda$ is called an $(r, s)$-fuzzy closed $((r, s)$-fc, for short) set iff $\underline{1}-\lambda$ is an $(r, s)$-fo set. Let $\left(X, \tau_{1}, \tau_{1}^{*}\right)$ and $\left(Y, \tau_{2}, \tau_{2}^{*}\right)$ be two $I$-dfts's. A function $\tilde{f}: X \rightarrow Y$ is said to be a double fuzzy continuous iff $\tau_{1}\left(f^{-1}(v)\right) \geq \tau_{2}(v)$ and $\tau_{1}^{*}\left(f^{-1}(v)\right) \leq \tau_{2}^{*}(v)$ for each $v \in I^{Y}$.

There was a question we must ask ourselve before starting to present our results, which was: Is it useful to introduce new concepts to I-double gradation fuzzy topological spaces?

We could know that double (initially, intuitionistic) fuzzy sets (and hence double fuzzy topological spaces) deal with ambiguity in a way better than fuzzy sets. In addition to that, double fuzzy topological spaces is a generalization of some other kinds of topological spaces; we can get fuzzy topological spaces in Chang's sense $\left(X, \mathcal{T}_{r, s}\right)$, where

$$
\mathcal{T}_{(r, s)}=\left\{\lambda \in I^{X} \mid \tau(\lambda) \geq r, \quad \tau^{*}(\lambda) \leq s\right\} .
$$

Also, when the conditions $\tau^{*}(\lambda)=1-\tau(\lambda)$ and $\tau(\lambda)+$ $\tau^{*}(\lambda) \nless 1$ achieved in Definition 2.1, we get the definition of fuzzy topological spaces in Kubiak- Šostak's sense Kubiak (1985); Šostak (1985). If we use $2^{X}$ instead of $I^{X}$, the resulting topological structure will be called double gradation fuzzifying topological spaces (A new structure mentioned for the first time in Bhaumik and Abbas 2008). Besides, we can also get the general topological spaces.

Theorem 2.1. [(Çoker and Demirci 1996; Lee and Im (2001)] Let $\left(X, \tau, \tau^{*}\right)$ be an I-dfts. Then for each $r \in I_{0}, s \in$ $I_{1}$ and $\lambda \in I^{X}$, we define an operator $C_{\tau, \tau^{*}}: I^{X} \times I_{0} \times I_{1} \rightarrow$ $I^{X}$ as follows:

$C_{\tau, \tau^{*}}(\lambda, r, s)=\bigwedge\left\{\mu \in I^{X} \mid \lambda \leq \mu, \tau(\underline{1}-\mu) \geq r, \tau^{*}(\underline{1}-\mu) \leq s\right\}$.

For $\lambda, \mu \in I^{X}, r_{1}, r_{2} \in I_{0}$ and $s_{1}, s_{2} \in I_{1}$, the operator $C_{\tau, \tau^{*}}$ satisfies the following statements:

(C1) $C_{\tau, \tau^{*}}(\underline{0}, r, s)=\underline{0}$,

(C2) $\lambda \leq C_{\tau, \tau^{*}}(\lambda, r, s)$,

(C3) $C_{\tau, \tau^{*}}(\lambda, r, s) \vee C_{\tau, \tau^{*}}(\mu, r, s)=C_{\tau, \tau^{*}}(\lambda \vee \mu, r, s)$,

(C4) $C_{\tau, \tau^{*}}\left(\lambda, r_{1}, s_{1}\right) \leq C_{\tau, \tau^{*}}\left(\lambda, r_{2}, s_{2}\right)$ if $r_{1} \leq r_{2}$ and $s_{1} \geq s_{2}$

(C5) $C_{\tau, \tau^{*}}\left(C_{\tau, \tau^{*}}(\lambda, r, s), r, s\right)=C_{\tau, \tau^{*}}(\lambda, r, s)$.

Theorem 2.2. [(Çoker and Demirci 1996; Lee and Im 2001)] Let $\left(X, \tau, \tau^{*}\right)$ be an I-dfts. Then for each $r \in I_{0}, s \in$
$I_{1}$ and $\lambda \in I^{X}$, we define an operator $I_{\tau, \tau^{*}}: I^{X} \times I_{0} \times I_{1} \rightarrow$ $I^{X}$ as follows:

$$
I_{\tau, \tau^{*}}(\lambda, r, s)=\bigvee\left\{\mu \in I^{X} \mid \mu \leq \lambda, \tau(\mu) \geq r, \tau^{*}(\mu) \leq s\right\} .
$$

For $\lambda, \mu \in I^{X}, r, r_{1}, r_{2} \in I_{0}$ and $s, s_{1}, s_{2} \in I_{1}$, the operator $I_{\tau, \tau} *$ satisfies the following statements:

(I1) $I_{\tau, \tau^{*}}(\underline{1}-\lambda, r, s)=\underline{1}-C_{\tau, \tau^{*}}(\lambda, r, s)$,

(I2) $I_{\tau, \tau^{*}}(\underline{1}, r, s)=\underline{1}$,

(I3) $I_{\tau, \tau^{*}}(\lambda, r, s) \leq \bar{\lambda}$,

(I4) $I_{\tau, \tau^{*}}(\lambda, r, s) \wedge I_{\tau, \tau^{*}}(\mu, r, s)=I_{\tau, \tau^{*}}(\lambda \wedge \mu, r, s)$,

(I5) $I_{\tau, \tau^{*}}\left(\lambda, r_{1}, s_{1}\right) \geq I_{\tau, \tau^{*}}\left(\lambda, r_{2}, s_{2}\right)$ if $r_{1} \leq r_{2}$ and $s_{1} \geq s_{2}$,

(I6) $I_{\tau, \tau^{*}}\left(I_{\tau, \tau^{*}}(\lambda, r, s), r, s\right)=I_{\tau, \tau^{*}}(\lambda, r, s)$,

(I7) If $I_{\tau, \tau^{*}}\left(C_{\tau, \tau^{*}}(\lambda, r, s), r, s\right)=\lambda$, then

$C_{\tau, \tau^{*}}\left(I_{\tau, \tau^{*}}(\underline{1}-\lambda, r, s), r, s\right)=\underline{1}-\lambda$.

Definition 2.2. Let $\left(X, \tau, \tau^{*}\right)$ be an I-dfts. For $\lambda \in I^{X}$, $r \in I_{0}$ and $s \in I_{1}$.

(1) $\lambda$ is called $(r, s)$-fuzzy preopen ( $(r, s)$-fpo, for short) if $\lambda \leq I_{\tau, \tau^{*}}\left(C_{\tau, \tau^{*}}(\lambda, r, s), r, s\right)$. A fuzzy set $\lambda$ is called $(r, s)$-fuzzy preclosed $((r, s)$-fpc, for short) iff $\underline{1}-\lambda$ is $(r, s)$-fpo set. The $(r, s)$-fuzzy preinterior of $\lambda$, denoted by $P I_{\tau, \tau^{*}}(\lambda, r, s)$ is defined by

$$
P I_{\tau, \tau^{*}}(\lambda, r, s)=\bigvee\left\{v \in I^{X} \mid v \leq \lambda, v \text { is }(r, s)-f p o\right\} .
$$

The $(r, s)$-fuzzy preclosure of $\lambda$, denoted by $P C_{\tau, \tau^{*}}(\lambda, r, s)$ is defined by

$$
P C_{\tau, \tau^{*}}(\lambda, r, s)=\bigwedge\left\{v \in I^{X} \mid \lambda \leq v, v \text { is }(r, s)-f p c\right\} .
$$

(2) $\lambda$ is called $(r, s)$-fuzzy regular open $((r, s)$-fro, for short) if $\lambda=I_{\tau, \tau^{*}}\left(C_{\tau, \tau^{*}}(\lambda, r, s), r, s\right)$. A fuzzy set $\lambda$ is called $(r, s)$-fuzzy regular closed $((r, s)$-frc, for short) iff $\underline{1}-\lambda$ is $(r, s)$-fro set.

(3) $\lambda$ is called $(r, s)$-fuzzy $\alpha$-open $((r, s)$-foo, for short) if $\lambda \leq I_{\tau, \tau^{*}}\left(C_{\tau, \tau^{*}}\left(I_{\tau, \tau^{*}}(\lambda, r, s), r, s\right), r, s\right)$. A fuzzy set $\lambda$ is called $(r, s)$-fuzzy $\alpha$-closed $((r, s)$-foc, for short) iff $\underline{1}-\lambda$ is $(r, s)$-foo set.

Theorem 2.3. Let $\left(X, \tau, \tau^{*}\right)$ be an I-dfts. For $\lambda \in I^{X}, r \in$ $I_{0}$ and $s \in I_{1}$.

(1) $\lambda$ is $(r, s)$-fpo (resp. $(r, s)$-fpc) iff $\lambda=P I_{\tau, \tau} *(\lambda, r, s)$ (resp. $\lambda=P C_{\tau, \tau^{*}}(\lambda, r, s)$ ),

(2) $I_{\tau, \tau^{*}}(\lambda, r, s) \leq P I_{\tau, \tau^{*}}(\lambda, r, s) \leq \lambda \leq P C_{\tau, \tau^{*}}(\lambda, r, s) \leq$ $C_{\tau, \tau^{*}}(\lambda, r, s)$

(3) $\underline{1}-P I_{\tau, \tau^{*}}(\lambda, r, s)=P C_{\tau, \tau^{*}}(\underline{1}-\lambda, r, s)$ and $P I_{\tau, \tau^{*}}(\underline{1}-\lambda, r, s)=\underline{1}-P C_{\tau, \tau^{*}}(\lambda, r, s)$.

Definition 2.3. Let $f:\left(X, \tau_{1}, \tau_{1}^{*}\right) \rightarrow\left(Y, \tau_{2}, \tau_{2}^{*}\right)$ be a function from an I-dfts $\left(X, \tau_{1}, \tau_{1}^{*}\right)$ into an I-dfts $\left(Y, \tau_{2}, \tau_{2}^{*}\right)$. The function $f$ is called: 
(1) double fuzzy preclosed if $f(\lambda)$ is $(r, s)$-fpc set in $I^{Y}$ for each $\lambda \in I^{X}, r \in I_{0}$ and $s \in I_{1} ; \tau_{1}(\underline{1}-\lambda) \geq r$, $\tau_{1}^{*}(\underline{1}-\lambda) \leq s$,

(2) double fuzzy open if $\tau_{2}(f(\lambda)) \geq \tau_{1}(\lambda)$ and $\tau_{2}^{*}(f(\lambda)) \leq \tau_{1}^{*}(\lambda)$ for each $\lambda \in I^{X}, r \in I_{0}$ and $s \in I_{1}$,

(3) double fuzzy almost open if $\tau_{2}(f(\lambda)) \geq r$ and $\tau_{2}^{*}(f(\lambda)) \leq s$ for each $(r, s)$-fro set $\lambda \in I^{X}, r \in I_{0}$ and $s \in I_{1}$.

Definition 2.4. Let $f:\left(X, \tau_{1}, \tau_{1}^{*}\right) \rightarrow\left(Y, \tau_{2}, \tau_{2}^{*}\right)$ be a function from an I-dfts $\left(X, \tau_{1}, \tau_{1}^{*}\right)$ into an I-dfts $\left(Y, \tau_{2}, \tau_{2}^{*}\right)$. The function $f$ is called:

(1) double fuzzy weakly open if $f(\lambda) \leq I_{\tau_{2}, \tau_{2}^{*}}\left(f\left(C_{\tau_{1}, \tau_{1}^{*}}(\lambda, r, s)\right), r, s\right)$ for each $\lambda \in I^{X}$, $r \in I_{0}$ and $s \in I_{1} ; \tau_{1}(\lambda) \geq r$ and $\tau_{1}^{*}(\lambda) \leq s$,

(2) double fuzzy $\alpha$-open if $\bar{f}(\lambda)$ is $(r, s)$-foo in $I^{Y}$ for each $\lambda \in I^{X}, r \in I_{0}$ and $s \in I_{1} ; \tau_{1}(\lambda) \geq r$ and $\tau_{1}^{*}(\lambda) \leq s$.

Definition 2.5. Let $\left(X, \tau, \tau^{*}\right)$ be an I-dfts, $\mu \in I^{X}, x_{t} \in$ $\mathbf{P}(X), r \in I_{0}$ and $s \in I_{1}$ where $\mathbf{P}(X)$ is the family of all fuzzy points in $X . \mu$ is called an $(r, s)$-fuzzy open $Q$-neighborhood of $x_{t}$ if $\tau(\mu) \geq r, \tau^{*}(\mu) \leq s$ and $x_{t} q \mu$. We denote the set of all $(r, s)$-fuzzy open Q-neighborhood of $x_{t}$ by $\mathbf{Q}_{\tau, \tau^{*}}\left(x_{t}, r, s\right)$.

Definition 2.6. Let $\left(X, \tau, \tau^{*}\right)$ be an I-dfts, $\lambda \in I^{X}, x_{t} \in$ $\mathbf{P}(X), r \in I_{0}$ and $s \in I_{1}$. $x_{t}$ is called $(r, s)$-fuzzy $\theta$ cluster point of $\lambda$ if for every $\mu \in Q_{\tau, \tau^{*}}\left(x_{t}, r, s\right)$, we have $C_{\tau, \tau^{*}}(\mu, r, s) q \lambda$. We denote $D_{\tau, \tau^{*}}(\lambda, r, s)=\bigvee\left\{x_{t} \in \boldsymbol{P}(X) \mid\right.$ $x_{t}$ is $(r, s)$-fuzzy $\theta$-cluster point of $\left.\lambda\right\}$. Where $D_{\tau, \tau^{*}}(\lambda, r, s)$ is called $(r, s)$-fuzzy $\theta$-closure of $\lambda$.

Theorem 2.4. Let $\left(X, \tau, \tau^{*}\right)$ an I-dfts. For $\lambda, \mu \in I^{X}$ and $r, s \in I_{0}$, we have the following:

(1) $D_{\tau, \tau^{*}}(\lambda, r, s)=\bigwedge\left\{\mu \in I^{X} \mid \lambda \leq\right.$ $\left.I_{\tau, \tau^{*}}(\mu, r, s), \tau(\underline{1}-\mu) \geq r, \tau^{*}(\underline{1}-\mu) \leq s\right\}$,

(2) $x_{t}$ is $(r, s)$-fuzzy $\theta$-cluster point of $\lambda$ iff $x_{t} \in D_{\tau, \tau^{*}}(\lambda, r, s)$.

(3) $C_{\tau, \tau^{*}}(\lambda, r, s) \leq D_{\tau, \tau^{*}}(\lambda, r, s)$,

(4) If $\tau(\lambda) \geq r$ and $\tau^{*}(\lambda) \leq s$, then $C_{\tau, \tau^{*}}(\lambda, r, s)=D_{\tau, \tau^{*}}(\lambda, r, s)$,

(5) If $\lambda$ is $(r, s)$-fpo, then $C_{\tau, \tau^{*}}(\lambda, r, s)=D_{\tau, \tau^{*}}(\lambda, r, s)$,

(6) If $\lambda$ is $(r, s)$-fpo and $\lambda=C_{\tau, \tau^{*}}\left(I_{\tau, \tau^{*}}(\lambda, r, s), r, s\right)$, then $D_{\tau, \tau^{*}}(\lambda, r, s)=\lambda$.

The complement of $(r, s)$-fuzzy $\theta$-closed set is called $(r, s)$-fuzzy $\theta$-open and the $(r, s)$-fuzzy $\theta$-interior operator denoted by $T_{\tau, \tau^{*}}(\lambda, r, s)$ is defined by $T_{\tau, \tau^{*}}(\lambda, r, s)=$ $\bigvee\left\{v \in I^{X} \mid C_{\tau, \tau^{*}}(v, r, s) \leq \lambda, \tau(v) \geq r, \tau^{*}(v) \leq s\right\}$.

Remark 2.1. From Theorem 2.4 It is easy to see that:

(1) $I_{\tau, \tau *}(\lambda, r, s) \leq T_{\tau, \tau^{*}}(\lambda, r, s)$ for any $\lambda \in I^{X}, r \in I_{0}$ and $s \in I_{1}$,
(2) $T_{\tau, \tau^{*}}(\lambda, r, s)=I_{\tau, \tau^{*}}(\lambda, r, s)$ for each $\lambda \in I^{X}, r \in I_{0}$ and $s \in I_{1} ; \tau(\lambda) \geq r$ and $\tau^{*}(\lambda) \leq s$.

\section{Double Fuzzy weakly preopen functions}

Definition 3.7. A function $f:\left(X, \tau_{1}, \tau_{1}^{*}\right) \rightarrow\left(Y, \tau_{2}, \tau_{2}^{*}\right)$ is said to be double fuzzy weakly preopen if

$$
f(\lambda) \leq P I_{\tau_{2}, \tau_{2}^{*}}\left(f\left(C_{\tau_{1}, \tau_{1}^{*}}(\lambda, r, s)\right), r, s\right)
$$

for each $\lambda \in I^{X}, r \in I_{0}$ and $s \in I_{1} ; \tau_{1}(\lambda) \geq r$ and $\tau_{1}^{*}(\lambda) \leq s$.

Remark 3.2. Every double fuzzy weakly open function is double fuzzy preopen and every double fuzzy preopen function is double fuzzy weakly preopen, but the converse need not be true in general.

Example 3.1. Let $X=\{a, b, c\}$ and $Y=\{x, y, z\}$. Fuzzy sets $\lambda_{1}, \lambda_{2}$ and $\lambda_{3}$ are defined as:

$$
\begin{array}{lll}
\lambda_{1}(a)=0.5, & \lambda_{1}(b)=0.3, & \lambda_{1}(c)=0.2, \\
\lambda_{2}(x)=0.9, & \lambda_{2}(y)=1, & \lambda_{2}(z)=0.7, \\
\lambda_{3}(x)=0.2, & \lambda_{3}(y)=0.2, & \lambda_{3}(z)=0.3 .
\end{array}
$$

Define $\tau_{1}$ and $\tau_{2}$ as follows:

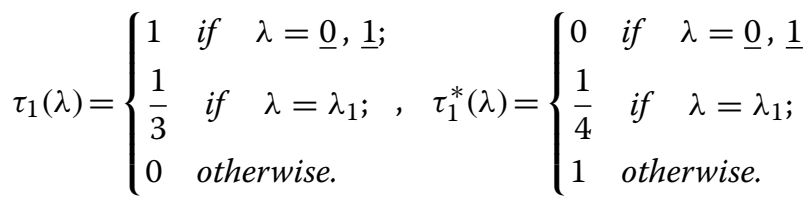

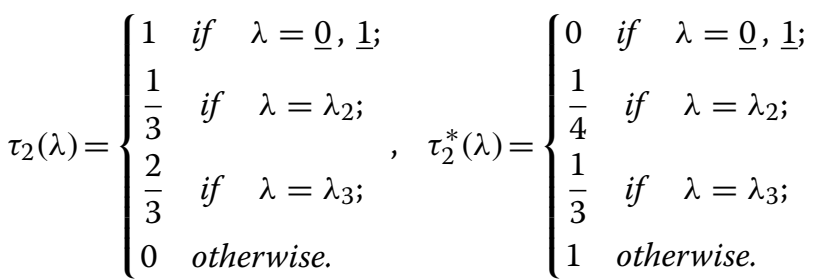

Then the mapping $f:\left(X, \tau_{1}, \tau_{1}^{*}\right) \rightarrow\left(Y, \tau_{2}, \tau_{2}^{*}\right)$ defined by $f(a)=z, f(b)=x$ and $f(c)=y$ is double fuzzy weakly preopen but not double fuzzy preopen. Where $\tau_{1}(\lambda) \geq \frac{1}{3}$, $\tau_{1}^{*}(\lambda) \leq \frac{1}{3}$ and $f(\lambda)$ is not $\left(\frac{1}{3}, \frac{1}{3}\right)$-fpo.

Example 3.2. Let $X=\{a, b, c\}$ and $Y=\{x, y, z\}$. Fuzzy sets $\lambda_{1}, \lambda_{2}$ and $\lambda_{3}$ are defined as:

$$
\begin{array}{lll}
\lambda_{1}(a)=0.5, & \lambda_{1}(b)=0.3, & \lambda_{1}(c)=0.2 ; \\
\lambda_{2}(x)=0.9, & \lambda_{2}(y)=1, & \lambda_{2}(z)=0.7 ; \\
\lambda_{3}(x)=0.2, & \lambda_{3}(y)=0.9, & \lambda_{3}(z)=0.3 .
\end{array}
$$

Let $\left(\tau_{1}, \tau_{1}^{*}\right)$ and $\left(\tau_{2}, \tau_{2}^{*}\right)$ defined as follows:

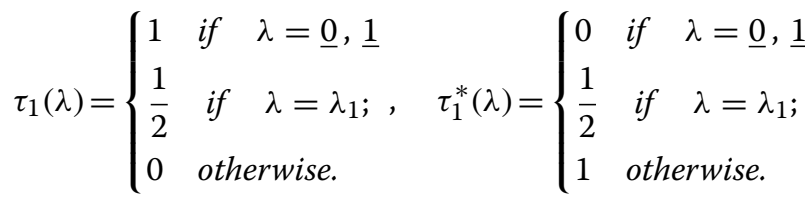




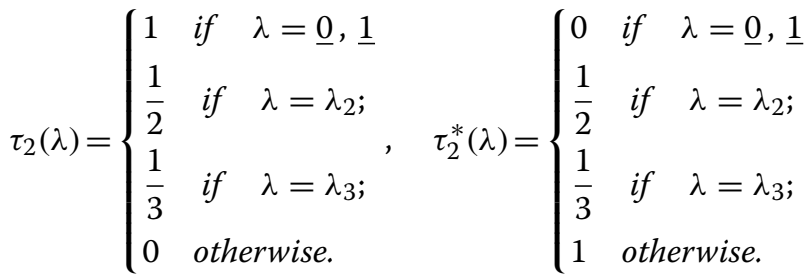

Then the mapping $f:\left(X, \tau_{1}, \tau_{1}^{*}\right) \rightarrow\left(Y, \tau_{2}, \tau_{2}^{*}\right)$ defined by $f(a)=z, f(b)=x$ and $f(c)=y$ is double fuzzy weakly preopen but not double fuzzy weakly open. Since $f\left(\lambda_{1}\right) \nless$ $I_{\tau_{2}, \tau_{2}^{*}}\left(f\left(C_{\tau_{1}, \tau_{1}^{*}}\left(\lambda_{1}, r, s\right)\right), r, s\right)$.

Theorem 3.5. For a function $f:\left(X, \tau_{1}, \tau_{1}^{*}\right) \rightarrow(Y$, $\left.\tau_{2}, \tau_{2}^{*}\right)$. The following statements are equivalent:

(1) $f$ is double fuzzy weakly preopen,

(2) $f\left(T_{\tau_{1}, \tau_{1}^{*}}(\lambda, r, s)\right) \leq P I_{\tau_{2}, \tau_{2}^{*}}(f(\lambda), r, s)$ for each $\lambda \in I^{X}$, $r \in I_{0}$ and $s \in I_{1}$,

(3) $T_{\tau_{1}, \tau_{1}^{*}}\left(f^{-1}(v), r, s\right) \leq f^{-1}\left(P I_{\tau_{2}, \tau_{2}^{*}}(v, r, s)\right)$ for each $v \in I^{Y}, r \in I_{0}$ and $s \in I_{1}$,

(4) $f^{-1}\left(P C_{\tau_{2}, \tau_{2}^{*}}(\nu, r, s)\right) \leq D_{\tau_{1}, \tau_{1}^{*}}\left(f^{-1}(\nu), r, s\right)$ for each $v \in I^{Y}, r \in I_{0}$ and $s \in I_{1}$.

Proof. (1) $\Rightarrow$ (2) Let $\lambda \in I^{X}$ and $x_{p} \in T_{\tau_{1}, \tau_{1}^{*}}(\lambda, r, s)$. Then there exists $\gamma \in \mathbf{Q}_{\tau_{1}, \tau_{1}^{*}}\left(x_{p}, r, s\right)$ such that $\gamma \leq$ $C_{\tau_{1}, \tau_{1}^{*}}(\gamma, r, s) \leq \lambda$. Thus $f(\gamma) \leq f\left(C_{\tau_{1}, \tau_{1}^{*}}(\gamma, r, s)\right) \leq f(\lambda)$ and hence

$$
\begin{aligned}
P I_{\tau_{2}, \tau_{2}^{*}}(f(\gamma), r, s) & \leq P I_{\tau_{2}, \tau_{2}^{*}}\left(f\left(C_{\tau_{1}, \tau_{1}^{*}}(\gamma, r, s)\right), r, s\right) \\
& \leq P I_{\tau_{2}, \tau_{2}^{*}}(f(\lambda), r, s) .
\end{aligned}
$$

Since $f$ is double fuzzy weakly preopen,

$$
f(\gamma) \leq P I_{\tau_{2}, \tau_{2}^{*}}\left(f\left(C_{\tau_{1}, \tau_{1}^{*}}(\gamma, r, s)\right), r, s\right) \leq P I_{\tau_{2}, \tau_{2}^{*}}(f(\lambda), r, s) .
$$

and hence $f\left(x_{p}\right) \in P I_{\tau_{2}, \tau_{2}^{*}}(f(\lambda), r, s)$. This shows that $x_{p} \in f^{-1}\left(P I_{\tau_{2}}(f(\lambda), r, s)\right)$. Thus $T_{\tau_{1}}(\lambda, r, s) \leq f^{-1}\left(P I_{\tau_{2}, \tau_{2}^{*}}\right.$ $(f(\lambda), r, s))$ and so, $f\left(T_{\tau_{1}, \tau_{1}^{*}}(\lambda, r, s)\right) \leq P I_{\tau_{2}, \tau_{2}^{*}}(f(\lambda), r, s)$.

(2) $\Rightarrow$ (1) Let $\mu \in I^{X}$; $\tau_{1}(\mu) \geq r$ and $\tau_{1}^{*}(\mu) \leq s$. Since $\mu \leq T_{\tau_{1}, \tau_{1}^{*}}\left(C_{\tau_{1}, \tau_{1}^{*}}(\mu, r, s), r, s\right)$, then

$$
\begin{aligned}
f(\mu) & \leq f\left(T_{\tau_{1}, \tau_{1}^{*}}\left(C_{\tau_{1}, \tau_{1}^{*}}(\mu, r, s), r, s\right)\right) \\
& \leq P I_{\tau_{2}, \tau_{2}^{*}}\left(f\left(C_{\tau_{1}, \tau_{1}^{*}}(\mu, r, s)\right), r, s\right) .
\end{aligned}
$$

Hence $f$ is double fuzzy weakly preopen.

(2) $\Rightarrow$ (3) Let $v \in I^{Y}$. By using (2), $f\left(T_{\tau_{1}, \tau_{1}^{*}}\left(f^{-1}(v)\right.\right.$, $r, s)) \leq P I_{\tau_{2}, \tau_{2}^{*}}(\nu, r, s)$. Therefore, $T_{\tau_{1}, \tau_{1}^{*}}\left(f^{-1}(\nu), r, s\right) \leq$ $f^{-1}\left(P I_{\tau_{2}, \tau_{2}^{*}}(v, r, s)\right)$.

(3) $\Rightarrow$ (2) Trivial
(3) $\Rightarrow$ (4) Let $v \in I^{Y}$. Using (3), we have

$$
\begin{aligned}
\underline{1}-D_{\tau_{1}, \tau_{1}^{*}}\left(f^{-1}(v), r, s\right) & =T_{\tau_{1}, \tau_{1}^{*}}\left(\underline{1}-f^{-1}(v), r, s\right) \\
& =T_{\tau_{1}, \tau_{1}^{*}}\left(f^{-1}(\underline{1}-v), r, s\right) \\
& \leq f^{-1}\left(P I_{\tau_{2}, \tau_{2}^{*}}(\underline{1}-v, r, s)\right) \\
& =f^{-1}\left(\underline{1}-P C_{\tau_{2}, \tau_{2}^{*}}(v, r, s)\right) \\
& =\underline{1}-\left(f^{-1}\left(P C_{\tau_{2}, \tau_{2}^{*}}(v, r, s)\right)\right) .
\end{aligned}
$$

Therefore, we obtain $f^{-1}\left(P C_{\tau_{2}, \tau_{2}^{*}}(\nu, r, s)\right) \leq D_{\tau_{1}, \tau_{1}^{*}}\left(f^{-1}(\nu)\right.$, $r, s)$.

(4) $\Rightarrow$ (3) Similarly we obtain, $\underline{1}-f^{-1}\left(P I_{\tau_{2}, \tau_{2}^{*}}(\nu, r, s)\right) \leq$ $\underline{1}-T_{\tau_{1}, \tau_{1}^{*}}\left(f^{-1}(v), r, s\right)$, for every $v \in I^{Y}, r \in I_{0}$ and $s \in I_{1}$, i.e., $T_{\tau_{1}, \tau_{1}^{*}}\left(f^{-1}(v), r, s\right) \leq f^{-1}\left(P I_{\tau_{2}, \tau_{2}^{*}}(v, r, s)\right)$.

Theorem 3.6. For the function $f:\left(X, \tau_{1}, \tau_{1}^{*}\right) \rightarrow(Y$, $\left.\tau_{2}, \tau_{2}^{*}\right)$. The following statements are equivalent:

(1) $f$ is double fuzzy weakly preopen,

(2) For each $x_{t} \in \mathbf{P}(X)$ and each $\mu \in I^{X}$; $\tau_{1}(\mu) \geq r$ and $\tau_{1}^{*}(\mu) \leq s$ with $x_{t} \leq \mu$, there exists $(r, s)$-fpo set $\gamma$ such that $f\left(x_{t}\right) \leq \gamma$ and $\gamma \leq f\left(C_{\tau_{1}, \tau_{1}^{*}}(\mu, r, s)\right)$.

Proof. (1) $\Rightarrow$ (2) Let $x_{t} \in \mathbf{P}(X)$ and $\mu \in I^{X}$ such that $\tau_{1}(\mu) \geq r, \tau_{1}^{*}(\mu) \leq s$ and $x_{t} \leq \mu$. Since $f$ is double fuzzy weakly preopen, then $f(\mu) \leq P I_{\tau_{2}, \tau_{2}^{*}}\left(f\left(C_{\tau_{1}, \tau_{1}^{*}}(\mu, r, s)\right), r, s\right)$. Let $\gamma=P I_{\tau_{2}, \tau_{2}^{*}}\left(f\left(C_{\tau_{1}, \tau_{1}^{*}}(\mu, r, s)\right), r, s\right)$. Hence $\gamma \leq f\left(C_{\tau_{1}, \tau_{1}^{*}}\right.$ $(\mu, r, s))$, with $f\left(x_{t}\right) \leq \gamma$.

(2) $\Rightarrow$ (1) Let $\mu \in I^{X} ; \tau_{1}(\mu) \geq r, \tau_{1}^{*}(\mu) \leq s$ and $y_{s} \leq f(\mu)$. It follows from (2) that $\gamma \leq f\left(C_{\tau_{1}, \tau_{1}^{*}}(\mu, r, s)\right)$ for some $(r, s)$-fpo $\gamma \in I^{Y}$ and $y_{s} \leq \gamma$. Hence we have, $y_{s} \leq \gamma \leq P I_{\tau_{2}, \tau_{2}^{*}}\left(f\left(C_{\tau_{1}, \tau_{1}^{*}}(\mu, r, s)\right), r, s\right)$. This shows that $f(\mu) \leq P I_{\tau_{2}, \tau_{2}^{*}}\left(f\left(C_{\tau_{1}, \tau_{1}^{*}}(\mu, r, s)\right), r, s\right)$, i.e. $f$ is double fuzzy weakly preopen function.

Theorem 3.7. Letf $:\left(X, \tau_{1}, \tau_{1}^{*}\right) \rightarrow\left(Y, \tau_{2}, \tau_{2}^{*}\right)$ be a bijective function. Then the following statements are equivalent:

(1) $f$ is double fuzzy weakly preopen;

(2) $P C_{\tau_{2}, \tau_{2}^{*}}(f(\lambda), r, s) \leq f\left(C_{\tau_{1}, \tau_{1}^{*}}(\lambda, r, s)\right)$ for each $\lambda \in I^{X}$, $r \in I_{0}$ and $s \in I_{1} ; \tau_{1}(\lambda) \geq r$ and $\tau_{1}^{*}(\lambda) \leq s$

(3) $P C_{\tau_{2}, \tau_{2}^{*}}\left(f\left(I_{\tau_{1}, \tau_{1}^{*}}(v, r, s)\right), r, s\right) \leq f(v)$ for each $v \in I^{X}$, $r \in I_{0}$ and $s \in I_{1} ; \tau_{1}(\underline{1}-v) \geq r$ and $\tau_{1}^{*}(\underline{1}-v) \leq s$.

Proof. (1) $\Rightarrow$ (2) Let $v \in I^{X} ; \tau_{1}(v) \geq r$ and $\tau_{1}^{*}(v) \leq s$. Then we have,

$$
f(\underline{1}-v)=\underline{1}-f(v) \leq P I_{\tau_{2}, \tau_{2}^{*}}\left(f\left(C_{\tau_{1}, \tau_{1}^{*}}(\underline{1}-v, r, s)\right), r, s\right),
$$

and so $\underline{1}-f(\nu) \leq \underline{1}-P C_{\tau_{2}, \tau_{2}^{*}}\left(f\left(I_{\tau_{1}, \tau_{1}^{*}}(\nu, r, s)\right), r, s\right)$. Hence $P C_{\tau_{2}, \tau_{2}^{*}}\left(f\left(I_{\tau_{1}, \tau_{1}^{*}}(\nu, r, s)\right), r, s\right) \leq f(\nu)$.

(2) $\Rightarrow$ (3) Let $\lambda \in I^{X} ; \tau_{1}(\lambda) \geq r$ and $\tau_{1}^{*}(\lambda) \leq s$. Since $C_{\tau_{1}, \tau_{1}^{*}}(\lambda, r, s)$ is $(r, s)$-fc set and $\lambda \leq I_{\tau_{1}, \tau_{1}^{*}}\left(C_{\tau_{1}, \tau_{1}^{*}}(\lambda, r, s)\right.$, 
$r, s)$ by (3) we have $P C_{\tau_{2}, \tau_{2}^{*}}(f(\lambda), r, s) \leq P C_{\tau_{2}, \tau_{2}^{*}}\left(f\left(I_{\tau_{1}, \tau_{1}^{*}}\right.\right.$ $(\lambda, r, s)), r, s) \leq f\left(C_{\tau_{1}, \tau_{1}^{*}}(\lambda, r, s)\right)$.

(3) $\Rightarrow$ (2) Trivial.

(2) $\Rightarrow$ (1) Trivial.

Theorem 3.8. For a function $f:\left(X, \tau_{1}, \tau_{1}^{*}\right) \rightarrow(Y$, $\left.\tau_{2}, \tau_{2}^{*}\right)$. The following statements are equivalent:

(1) $f$ is double fuzzy weakly preopen;

(2) $f\left(I_{\tau_{1}, \tau_{1}^{*}}(v, r, s)\right) \leq P I_{\tau_{2}, \tau_{2}^{*}}(f(v), r, s)$ for each $v \in I^{X}$, $r \in I_{0}$ and $s \in I_{1} ; \tau_{1}(v) \geq r$ and $\tau_{1}^{*}(v) \leq s$;

(3) $f\left(I_{\tau_{1}, \tau_{1}^{*}}\left(C_{\tau_{1}, \tau_{1}^{*}}(\lambda, r, s), r, s\right)\right) \leq$ $P I_{\tau_{2}, \tau_{2}^{*}}\left(f\left(C_{\tau_{1}, \tau_{1}^{*}}(\lambda, r, s)\right), r, s\right)$ for each $\lambda \in I^{X}, r \in I_{0}$ and $s \in I_{1} ; \tau_{1}(\lambda) \geq r$ and $\tau_{1}^{*}(\lambda) \leq s$;

(4) $f(\lambda) \leq P I_{\tau_{2}, \tau_{2}^{*}}\left(f\left(C_{\tau_{1}, \tau_{1}^{*}}(\lambda, r, s)\right), r, s\right)$, for each $(r, s)$-fpo set $\lambda \in I^{X}$

(5) $f(\lambda) \leq P I_{\tau_{2}, \tau_{2}^{*}}\left(f\left(C_{\tau_{1}, \tau_{1}^{*}}(\lambda, r, s)\right), r, s\right)$, for each $(r, s)$-foo set $\lambda \in I^{X}$.

Proof. (1) $\Rightarrow$ (2) Let $v \in I^{X}, r \in I_{0}$ and $s \in I_{1} ; \tau_{1}(\underline{1}-v) \geq$ $r$ and $\tau_{1}^{*}(\underline{1}-v) \leq s$. By $(1)$,

$$
\begin{aligned}
f\left(I_{\tau_{1}, \tau_{1}^{*}}(\nu, r, s)\right) & \leq P I_{\tau_{2}, \tau_{2}^{*}}\left(f\left(C_{\tau_{1}, \tau_{1}^{*}}\left(I_{\tau_{1}, \tau_{1}^{*}}(\nu, r, s), r, s\right)\right), r, s\right) \\
& \leq P I_{\tau_{2}, \tau_{2}^{*}}\left(f\left(C_{\tau_{1}, \tau_{1}^{*}}(\nu, r, s)\right), r, s\right) \\
& =P I_{\tau_{2}, \tau_{2}^{*}}(f(v), r, s)
\end{aligned}
$$

(2) $\Rightarrow$ (3) It is clear.

(3) $\Rightarrow(4)$ Let $\lambda$ be $(r, s)$-fpo set. Hence by (3),

$$
\begin{aligned}
f(\lambda) & \leq f\left(I_{\tau_{1}, \tau_{1}^{*}}\left(C_{\tau_{1}, \tau_{1}^{*}}(\lambda, r, s), r, s\right)\right) \\
& \leq P I_{\tau_{2}, \tau_{2}^{*}}\left(f\left(C_{\tau_{1}, \tau_{1}^{*}}(\lambda, r, s)\right), r, s\right) . \\
(4) \Rightarrow & \text { (5) and }(5) \Rightarrow \text { (1) are clear. }
\end{aligned}
$$

Definition 3.8. A function $f:\left(X, \tau_{1}, \tau_{1}^{*}\right) \rightarrow\left(Y, \tau_{2}, \tau_{2}^{*}\right)$ is said to be double fuzzy strongly continuous, if $f\left(C_{\tau_{1}, \tau_{1}^{*}}(\lambda, r, s)\right) \leq f(\lambda)$ for each $\lambda \in I^{X}, r \in I_{0}$ and $s \in I_{1}$.

Theorem 3.9. If $f:\left(X, \tau_{1}, \tau_{1}^{*}\right) \rightarrow\left(Y, \tau_{2}, \tau_{2}^{*}\right)$ is double fuzzy weakly preopen and double fuzzy strongly continuous function, then $f$ is double fuzzy preopen.

Proof. Let $\lambda \in I^{X}$ such that $\tau_{1}(\lambda) \geq r$ and $\tau_{1}^{*}(\lambda) \leq s$. Since $f$ is double fuzzy weakly preopen

$$
f(\lambda) \leq P I_{\tau_{2}, \tau_{2}^{*}}\left(f\left(C_{\tau_{1}, \tau_{1}^{*}}(\lambda, r, s)\right), r, s\right) .
$$

However, since $f$ is double fuzzy strongly continuous, then $f(\lambda) \leq P I_{\tau_{2}, \tau_{2}^{*}}(f(\lambda), r, s)$ and therefore $f(\lambda)$ is $(r, s)$ fpo.

Definition 3.9. A function $f:\left(X, \tau_{1}, \tau_{1}^{*}\right) \rightarrow\left(Y, \tau_{2}, \tau_{2}^{*}\right)$ is said to be double fuzzy contra-preclosed if $f(\lambda)$ is $(r, s)$ fpo for each $\lambda \in I^{X}, r \in I_{0}$ and $s \in I_{1} ; \tau_{1}(\underline{1}-\lambda) \geq r$ and $\tau_{1}^{*}(\underline{1}-\lambda) \leq s$.
Theorem 3.10. If $f:\left(X, \tau_{1}, \tau_{1}^{*}\right) \rightarrow\left(Y, \tau_{2}, \tau_{2}^{*}\right)$ is double fuzzy contra-preclosed, then $f$ is double fuzzy weakly preopen function.

Proof. Let $\lambda \in I^{X} ; \tau_{1}(\lambda) \geq r$ and $\tau_{1}^{*}(\lambda) \leq s$. Then, we have

$$
f(\lambda) \leq f\left(C_{\tau_{1}, \tau_{1}^{*}}(\lambda, r, s)\right)=P I_{\tau_{2}, \tau_{2}^{*}}\left(f\left(C_{\tau_{1}, \tau_{1}^{*}}(\lambda, r, s)\right), r, s\right) .
$$

The converse of the above theorem need not be true in general as in the following Example.

Example 3.3. Let $X=\{a, b, c\}$ and $Y=\{x, y, z\}$. Define fuzzy sets $\lambda_{1}, \lambda_{2}$ as follows:

$$
\begin{aligned}
& \lambda_{1}(a)=0, \quad \lambda_{1}(b)=0.2, \quad \lambda_{1}(c)=0.7 ; \\
& \lambda_{2}(x)=0, \quad \lambda_{2}(y)=0.2, \quad \lambda_{2}(x)=0.2 \text {. }
\end{aligned}
$$

Let $\left(\tau_{1}, \tau_{1}^{*}\right)$ and $\left(\tau_{2}, \tau_{2}^{*}\right)$ defined as follows:

$\tau_{1}(\lambda)=\left\{\begin{array}{l}1 \quad \text { if } \lambda=\underline{1}, \underline{0} ; \\ \frac{1}{3} \quad \text { if } \lambda=\lambda_{1} ; ， \quad \tau_{1}^{*}(\lambda)=\left\{\begin{array}{ll}0 & \text { if } \lambda=\underline{1}, \underline{0} ; \\ 0 & \text { otherwise. }\end{array} \quad \text { if } \lambda=\lambda_{1} ;\right. \\ 1 \quad \text { otherwise. }\end{array}\right.$

$\tau_{2}(\lambda)=\left\{\begin{array}{l}1 \quad \text { if } \lambda=\underline{1}, \underline{0} ; \\ \frac{1}{3} \quad \text { if } \lambda=\lambda_{2} ;, \quad \tau_{2}^{*}(\lambda)=\left\{\begin{array}{ll}0 & \text { if } \lambda=\underline{1}, \underline{0} ; \\ 0 & \text { otherwise. }\end{array} \quad \text { if } \lambda=\lambda_{2} ;\right. \\ 1 \quad \text { otherwise. }\end{array}\right.$

Then the function $f:\left(X, \tau_{1}, \tau_{1}^{*}\right) \rightarrow\left(Y, \tau_{2}, \tau_{2}^{*}\right)$ defined as $f(a)=x, f(b)=y$ and $f(c)=z$ is double fuzzy weakly preopen but it isn't double fuzzy contra-preclosed.

Definition 3.10. An I-dfts $\left(X, \tau, \tau^{*}\right)$ is said to be $(r, s)$ fuzzy regular space if for each $\lambda \in I^{X} ; \tau(\lambda) \geq r$ and $\tau^{*}(\lambda) \leq s$ is a union of $(r, s)$-fo sets $\mu_{i} \in I^{X}$ such that $C_{\tau, \tau^{*}}\left(\mu_{i}, r, s\right) \leq \lambda$ for each $i \in J$.

Theorem 3.11. Let $\left(X, \tau, \tau^{*}\right)$ be $(r, s)$-regular fuzzy topological space. Then, $f:\left(X, \tau_{1}, \tau_{1}^{*}\right) \rightarrow\left(Y, \tau_{2}, \tau_{2}^{*}\right)$ is double fuzzy weakly preopen if and only iff is double fuzzy preopen.

Proof. The sufficiency is clear. For the necessity, let $\lambda \in$ $I^{X}, r \in I_{0}, s \in I_{1} ; \lambda \neq \underline{0}, \tau_{1}(\lambda) \geq r$ and $\tau_{1}^{*}(\lambda) \leq s$. For each $x_{t} \leq \lambda$, let $x_{t} \leq \mu_{x_{t}} \leq C_{\tau_{1}, \tau_{1}^{*}}\left(\mu_{x_{t}}, r, s\right) \leq \lambda$. Hence we 
obtain that $\lambda=\bigvee\left\{\mu_{x_{t}} \mid x_{t} \leq \lambda\right\}=\bigvee\left\{C_{\tau_{1}, \tau_{1}^{*}}\left(\mu_{x_{t}}, r, s\right) \mid\right.$ $\left.x_{t} \leq \lambda\right\}$ and,

$$
\begin{aligned}
f(\lambda) & =\bigvee\left\{f\left(\mu_{x_{t}}\right) \mid x_{t} \leq \lambda\right\} \\
& \leq \bigvee\left\{P I_{\tau_{2}, \tau_{2}^{*}}\left(f\left(C_{\tau_{1}, \tau_{1}^{*}}\left(\mu_{x_{t}}, r, s\right)\right), r, s\right) \mid x_{t} \leq \lambda\right\} \\
& \leq P I_{\tau_{2}, \tau_{2}^{*}}\left(f\left(\bigvee\left\{C_{\tau_{1}, \tau_{1}^{*}}\left(\mu_{x_{t}}, r, s\right) \mid x_{t}\right\}\right), r, s\right) \\
& =P I_{\tau_{2}, \tau_{2}^{*}}(f(\lambda), r, s) .
\end{aligned}
$$

Thus $f$ is double fuzzy preopen.

Theorem 3.12. Iff $:\left(X, \tau_{1}, \tau_{1}^{*}\right) \rightarrow\left(Y, \tau_{2}, \tau_{2}^{*}\right)$ is double fuzzy almost open function, then it is double fuzzy weakly preopen.

Proof. Let $\lambda \in I^{X} ; \tau_{1}(\lambda) \geq r$ and $\tau_{1}^{*}(\lambda) \leq s$. Since $f$ is double fuzzy almost open and $I_{\tau_{1}, \tau_{1}^{*}}\left(C_{\tau_{1}, \tau_{1}^{*}}(\lambda, r, s), r, s\right)$ is $(r, s)$-fro, then

$$
\begin{aligned}
I_{\tau_{2}, \tau_{2}^{*}}\left(f\left(I_{\tau_{1}, \tau_{1}^{*}}\left(C_{\tau_{1}, \tau_{1}^{*}}(\lambda, r, s), r, s\right)\right), r, s\right)= & f\left(I _ { \tau _ { 1 } , \tau _ { 1 } ^ { * } } \left(C_{\tau_{1}, \tau_{1}^{*}}\right.\right. \\
& \times(\lambda, r, s), r, s))
\end{aligned}
$$

and hence

$$
\begin{aligned}
f(\lambda) & \leq f\left(I_{\tau_{1}, \tau_{1}^{*}}\left(C_{\tau_{1}, \tau_{1}^{*}}(\lambda, r, s), r, s\right)\right. \\
& \leq I_{\tau_{2}, \tau_{2}^{*}}\left(f\left(C_{\tau_{1}, \tau_{1}^{*}}(\lambda, r, s)\right), r, s\right) \\
& \leq P I_{\tau_{2}, \tau_{2}^{*}}\left(f\left(C_{\tau_{1}, \tau_{1}^{*}}(\lambda, r, s)\right), r, s\right) .
\end{aligned}
$$

This shows that $f$ is double fuzzy weakly preopen.

Definition 3.11. Let $\left(X, \tau, \tau^{*}\right)$ be an I-dfts, $r \in I_{0}$ and $s \in I_{1}$. The two fuzzy sets $\lambda, \mu \in I^{X}$ are said to be $(r, s)$ fuzzy separated iff $\lambda \bar{q} C_{\tau, \tau^{*}}(\mu, r, s)$ and $\mu \bar{q} C_{\tau, \tau^{*}}(\lambda, r, s)$. A fuzzy set which cannot be expressed as a union of two $(r, s)$ fuzzy separated sets is said to be $(r, s)$-fuzzy connected.

Definition 3.12. Let $\left(X, \tau, \tau^{*}\right)$ an I-dfts. The fuzzy sets $\lambda, \mu \in I^{X}$ such that $\lambda \neq \underline{0}, \mu \neq \underline{0}$, are said to be fuzzy $(r, s)$ pre-separated if $\lambda \bar{q} P C_{\tau, \tau^{*}}(\mu, r, s)$ and $\mu \bar{q} P C_{\tau, \tau^{*}}(\lambda, r, s)$ or equivalently if there exist two $(r, s)$-fpo sets $v, \gamma$ such that $\lambda \leq \nu, \mu \leq \gamma, \lambda \bar{q} \gamma$ and $\mu \bar{q} \nu$. An I-dfts which can not be expressed as a union of two fuzzy $(r, s)$-pre-separated sets is said to be fuzzy $(r, s)$-pre-connected space.

Theorem 3.13. If $f:\left(X, \tau_{1}, \tau_{1}^{*}\right) \rightarrow\left(Y, \tau_{2}, \tau_{2}^{*}\right)$ is an injective double fuzzy weakly preopen and strongly double fuzzy continuous function from the space $\left(X, \tau_{1}, \tau_{1}^{*}\right)$ onto an $(r, s)$-fuzzy pre-connected space $\left(Y, \tau_{2}, \tau_{2}^{*}\right)$, then $\left(X, \tau_{1}, \tau_{1}^{*}\right)$ is $(r, s)$-fuzzy connected.

Proof. Let $\left(X, \tau_{1}, \tau_{1}^{*}\right)$ be not $(r, s)$-fuzzy connected. Then there exist $(r, s)$-fuzzy separated sets $\beta, \gamma \in I^{X}$ such that $\beta \vee \gamma=\underline{1}$. Since $\beta$ and $\gamma$ are $(r, s)$-fuzzy separated, there exists $\lambda, \mu \in I^{X} ; \tau_{1}(\lambda) \geq r, \tau_{1}(\mu) \geq r$ and $\tau_{1}^{*}(\lambda) \leq s, \tau_{1}^{*}(\mu) \leq s$ such that $\beta \leq \lambda, \gamma \leq \mu$, $\beta \bar{q} \mu$ and $\gamma \bar{q} \lambda$. Hence we have $f(\beta) \leq f(\lambda), f(\gamma) \leq$ $f(\mu), f(\beta) \bar{q} f(\mu)$ and $f(\gamma) \bar{q} f(\lambda)$. Since $f$ is double fuzzy weakly preopen and double fuzzy strongly continuous function, from Theorem 3.10 we have $f(\lambda)$ and $f(\mu)$ are $(r, s)$-fpo sets. Therefore, $f(\beta)$ and $f(\gamma)$ are $(r, s)$-fuzzy pre-separated and

$$
\underline{1}=f(\underline{1})=f(\beta \vee \gamma)=f(\beta) \vee f(\gamma)
$$

which is contradiction with $\left(Y, \tau_{2}, \tau_{2}^{*}\right)$ is $(r, s)$-fuzzy preconnected. Thus $\left(X, \tau_{1}, \tau_{1}^{*}\right)$ is $(r, s)$-fuzzy connected.

\section{Double Fuzzy weakly preclosed functions}

Definition 4.13. A function $f:\left(X, \tau_{1}, \tau_{1}^{*}\right) \rightarrow\left(Y, \tau_{2}, \tau_{2}^{*}\right)$ is said to be double fuzzy weakly preclosed function if

$$
P C_{\tau_{2}, \tau_{2}^{*}}\left(f\left(I_{\tau_{1}, \tau_{1}^{*}}(\lambda, r, s)\right), r, s\right) \leq f(\lambda)
$$

for each $\lambda \in I^{X}, r \in I_{0}$ and $s \in I_{1} ; \tau_{1}(\underline{1}-\lambda) \geq r$ and $\tau_{1}^{*}(\underline{1}-\lambda) \leq s$.

Remark 4.3. Clearly, every double fuzzy preclosed function is double fuzzy weakly preclosed, but the converse need not be true in general, as the next example shows.

Example 4.4. Let $X=\{a, b\}$ and $Y=\{x, y\}$. Fuzzy sets $\lambda_{1}$ and $\lambda_{2}$ are defined as:

$$
\begin{array}{ll}
\lambda_{1}(x)=0.4, & \lambda_{1}(y)=0.3 ; \\
\lambda_{2}(a)=0.5, & \lambda_{2}(b)=0.6 .
\end{array}
$$

Let

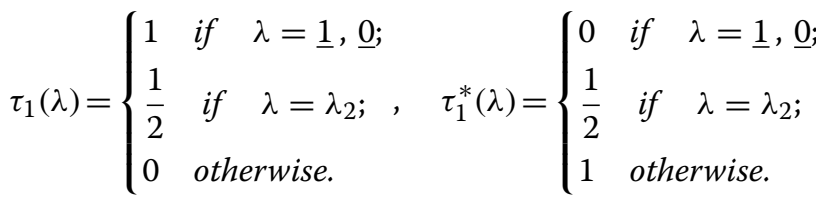

$\tau_{2}(\lambda)=\left\{\begin{array}{l}1 \quad \text { if } \lambda=\underline{1}, \underline{0} ; \\ \frac{1}{2} \quad \text { if } \lambda=\lambda_{1} ; \quad, \quad \tau_{2}^{*}(\lambda)=\left\{\begin{array}{ll}0 & \text { if } \lambda=\underline{1}, \underline{0} ; \\ 0 & \text { otherwise. }\end{array} \text { if } \lambda=\lambda_{1} ;\right. \\ \frac{1}{2} \text { otherwise. }\end{array}\right.$

Then the function $f:\left(X, \tau_{1}, \tau_{1}^{*}\right) \rightarrow\left(Y, \tau_{2}, \tau_{2}^{*}\right)$ defined by $f(a)=x, f(b)=y$ is double fuzzy weakly preclosed but is not double fuzzy preclosed.

Theorem 4.14. For a function $f:\left(X, \tau_{1}, \tau_{1}^{*}\right) \rightarrow$ $\left(Y, \tau_{2}, \tau_{2}^{*}\right)$. The following statements are equivalent.

(1) $f$ is double fuzzy weakly preclosed;

(2) $P C_{\tau_{2}, \tau_{2}^{*}}(f(\lambda), r, s) \leq f\left(C_{\tau_{1}, \tau_{1}^{*}}(\lambda, r, s)\right)$ for each $\lambda \in I^{X}$, $r \in I_{0}$ and $s \in I_{1} ; \tau_{1}(\lambda) \geq r$ and $\tau_{1}^{*}(\lambda) \leq s$;

(3) $P C_{\tau_{2}, \tau_{2}^{*}}\left(f\left(I_{\tau_{1}, \tau_{1}^{*}}(\lambda, r, s), r, s\right) \leq f(\lambda)\right.$ for each $\lambda \in I^{X}$, $r \in I_{0}$ and $s \in I_{1} ; \tau_{1}(\underline{1}-\lambda) \geq r$ and $\tau_{1}^{*}(\underline{1}-\lambda) \leq s$; 
(4) $P C_{\tau_{2}, \tau_{2}^{*}}\left(f\left(I_{\tau_{1}, \tau_{1}^{*}}(\lambda, r, s), r, s\right) \leq f(\lambda)\right.$ for each $(r, s)$-fpc set $\lambda \in I^{X}, r \in I_{0}$ and $s \in I_{1}$;

(5) $P C_{\tau_{2}, \tau_{2}^{*}}\left(f\left(I_{\tau_{1}, \tau_{1}^{*}}(\lambda, r, s), r, s\right) \leq f(\lambda)\right.$ for each $(r, s)$-foc $\lambda \in I^{X}, r \in I_{0}$ and $s \in I_{1}$.

Proof. Straightforward.

Theorem 4.15. For a function $f:\left(X, \tau_{1}, \tau_{1}^{*}\right) \rightarrow(Y$, $\left.\tau_{2}, \tau_{2}^{*}\right)$. The following statements are equivalent.

(1) $f$ is double fuzzy weakly preclosed;

(2) $P C_{\tau_{2}, \tau_{2}^{*}}(f(\lambda), r, s) \leq f\left(C_{\tau_{1}, \tau_{1}^{*}}(\lambda, r, s)\right)$ for each $(r, s)$-fro set $\lambda \in I^{X}, r \in I_{0}$ and $s \in I_{1}$

(3) For each $v \in I^{Y}, \mu \in I^{X}, r \in I_{0}$ and $s \in I_{1} ; \tau_{1}(\mu) \geq r$ and $\tau_{1}^{*}(\mu) \leq s$ with $f^{-1}(v) \leq \mu$, there exists $(r, s)$-fpo set $\gamma \in I^{Y}$ with $v \leq \gamma$ and $f^{-1}(\gamma) \leq C_{\tau_{1}, \tau_{1}^{*}}(\mu, r, s)$;

(4) For each fuzzy point $y_{s} \in \mathbf{P}(Y)$ and each $\mu \in I^{X}$, $r \in I_{0}$ and $s \in I_{1}$ such that $\tau_{1}(\mu) \geq r$ and $\tau_{1}^{*}(\mu) \leq s$ with $f^{-1}\left(y_{s}\right) \leq \mu$, there exists $(r, s)$-fpo set $\gamma \in I^{Y}$; $y_{s} \leq \gamma$ and $f^{-1}(\gamma) \leq C_{\tau_{1}, \tau_{1}^{*}}(\mu, r, s)$

(5) $P C_{\tau_{2}, \tau_{2}^{*}}\left(f\left(I_{\tau_{1}, \tau_{1}^{*}}\left(C_{\tau_{1}, \tau_{1}^{*}}(\lambda, r, s), r, s\right)\right), r, s\right) \leq$ $f\left(C_{\tau_{1}, \tau_{1}^{*}}(\lambda, r, s)\right)$ for each $\lambda \in I^{X}, r \in I_{0}$ and $s \in I_{1}$;

(6) $P C_{\tau_{2}, \tau_{2}^{*}}\left(f\left(I_{\tau_{1}, \tau_{1}^{*}}\left(D_{\tau_{1}, \tau_{1}^{*}}(\lambda, r, s), r, s\right)\right), r, s\right) \leq$ $f\left(D_{\tau_{1}, \tau_{1}^{*}}(\lambda, r, s)\right)$ for each $\lambda \in I^{X}, r \in I_{0}$ and $s \in I_{1}$;

(7) $P C_{\tau_{2}, \tau_{2}^{*}}(f(\lambda), r, s) \leq f\left(C_{\tau_{1}, \tau_{1}^{*}}(\lambda, r, s)\right)$ for each $(r, s)$-fpo set $\lambda \in I^{X}, r \in I_{0}$ and $s \in I_{1}$.

Proof. We will prove (2) $\Rightarrow$ (3) and (1) $\Rightarrow$ (6).

(2) $\Rightarrow$ (3) : Let $v \in I^{Y}, r \in I_{0}, s \in I_{1}$ and let $\mu \in I^{X} ; \tau_{1}(\mu) \geq r$ and $\tau_{1}^{*}(\mu) \leq s$ with $f^{-1}(\nu) \leq \mu$. Then $f^{-1}(\nu) \bar{q} C_{\tau_{1}, \tau_{1}^{*}}\left(\underline{1}-C_{\tau_{1}, \tau_{1}^{*}}(\mu, r, s), r, s\right)$ and consequently, $\nu \bar{q} f\left(C_{\tau_{1}, \tau_{1}^{*}}\left(\underline{1}-C_{\tau_{1}, \tau_{1}^{*}}(\mu, r, s), r, s\right)\right.$. Since $\underline{1}-C_{\tau_{1}, \tau_{1}^{*}}(\mu, r, s)$ is $(r, s)$-fro, $v \bar{q} P C_{\tau_{2}, \tau_{2}^{*}}\left(f\left(\underline{1}-C_{\tau_{1}, \tau_{1}^{*}}(\mu, r, s)\right), r, s\right)$ by $(2)$. Let $\gamma=\underline{1}-P C_{\tau_{2}, \tau_{2}^{*}}\left(f\left(\underline{1}-C_{\tau_{1}, \tau_{1}^{*}}(\mu, r, s)\right), r, s\right)$. Then $\gamma$ is $(r, s)$-fpo with $v \leq \gamma$ and

$$
\begin{aligned}
f^{-1}(\gamma) & \leq \underline{1}-f^{-1}\left(P C_{\tau_{2}, \tau_{2}^{*}}\left(\underline{1}-C_{\tau_{1}, \tau_{1}^{*}}(\mu, r, s), r, s\right)\right) \\
& \leq \underline{1}-f^{-1} f\left(\underline{1}-C_{\tau_{1}, \tau_{1}^{*}}(\mu, r, s)\right) \\
& \leq C_{\tau_{1}, \tau_{1}^{*}}(\mu, r, s) .
\end{aligned}
$$

(1) $\Rightarrow(6):$ Let $v \in I^{Y}, r \in I_{0}$ and $s \in I_{1} ; \tau_{2}(\underline{1}-v) \geq r$, $\tau_{2}^{*}(\underline{1}-v) \leq s$ and $y_{s} \leq \underline{1}-f(v)$. Since $f^{-1}\left(y_{s}\right) \leq$ $\underline{1}-v$, there exists $(r, s)$-fpo $\gamma \in I^{Y}$ with $y_{s} \leq \bar{\gamma}$ and $f^{-1}(\gamma) \leq C_{\tau_{1}, \tau_{1}^{*}}(\underline{1}-\nu, r, s)=\underline{1}-I_{\tau_{1}, \tau_{1}^{*}}(\nu, r, s)$ by (6). Therefore $\gamma \bar{q} f\left(I_{\tau_{1}, \tau_{1}^{*}}(v, r)\right)$, so that $y_{s} \leq \underline{1}-$ $P C_{\tau_{2}, \tau_{2}^{*}}\left(f\left(I_{\tau_{1}, \tau_{1}^{*}}(\nu, r, s)\right), r, s\right)$.

Theorem 4.16. Iff $:\left(X, \tau_{1}, \tau_{1}^{*}\right) \rightarrow\left(Y, \tau_{2}, \tau_{2}^{*}\right)$ is double fuzzy weakly preclosed, then for each $y_{s} \in \mathbf{P}(Y)$ and each $\mu \in \mathbf{Q}_{\tau_{1}, \tau_{1}^{*}}\left(f^{-1}\left(y_{s}\right), r, s\right)$, there exists $(r, s)$-fpo set $\gamma \in I^{Y}$; $\gamma \in \mathbf{Q}_{\tau_{2}, \tau_{2}^{*}}\left(y_{s}, r, s\right)$, such that $f^{-1}(\gamma) \leq C_{\tau_{1}, \tau_{1}^{*}}(\mu, r, s)$.

Proof. Let $\mu \in \mathbf{Q}_{\tau_{1}, \tau_{1}^{*}}\left(f^{-1}\left(y_{s}, r, s\right)\right.$. Then $\mu(x)+s>1$ and hence there exists $t \in(0,1)$ such that $\mu(x)>t>$ $1-s$. Then $\mu \in \mathbf{Q}_{\tau_{1}, \tau_{1}^{*}}\left(f^{-1}\left(y_{t}\right), r, s\right)$. By Theorem 3.76 there exists $(r, s)$-fpo set $\gamma \in I^{Y} ; y_{t} \leq \gamma$ such that $f^{-1}(\gamma) \leq C_{\tau_{1}, \tau_{1}^{*}}(\mu, r, s)$. Now, $\gamma(y)>t$ and hence $\gamma(y)>1-s$. Thus $\gamma$ is $(r, s)$-fpo neighborhood of $y_{s}$.

Definition 4.14. Let $\left(X, \tau, \tau^{*}\right)$ be an I-dfts. A fuzzy set $\lambda \in I^{X}$ is called $(r, s)$-fuzzy pre-Q-neighborhood of $x_{t}$ if there exists $(r, s)$-fpo set $\mu \in I^{X}$ such that $x_{t} q \mu \leq \lambda$. We denote the set of all $(r, s)$-fuzzy pre-Q-neighborhood of $x_{t}$ by $\mathbf{P} \boldsymbol{Q}_{\tau, \tau^{*}}\left(x_{t}, r, s\right)$.

Theorem 4.17. In an I-dfts $\left(X, \tau, \tau^{*}\right)$. A fuzzy point $x_{t} \in$ $P C_{\tau, \tau^{*}}(\lambda, r)$ if and only iffor every $\mu \in P Q_{\tau, \tau^{*}}\left(x_{t}, r, s\right), \mu q \lambda$ is hold.

Proof. Straightforward.

Theorem 4.18. Iff $:\left(X, \tau_{1}, \tau_{1}^{*}\right) \rightarrow\left(Y, \tau_{2}, \tau_{2}^{*}\right)$ is double fuzzy weakly preclosed and if for each $v \in I^{X}, r \in I_{0}$ and $s \in I_{1} ; \tau_{1}(\underline{1}-v) \geq r, \tau_{1}^{*}(\underline{1}-v) \leq$ s and each $f^{-1}\left(y_{s}\right) \leq \underline{1}-v$ there exists $\mu \in Q_{\tau_{1}, \tau_{1}^{*}}\left(f^{-1}\left(y_{s}\right), r, s\right)$ such that $f^{-1}\left(y_{s}\right) \leq$ $\mu \leq C_{\tau_{1}, \tau_{1}^{*}}(\mu, r, s) \leq \underline{1}-v$. Then $f$ is double fuzzy preclosed.

Proof. Let $v \in I^{X}, r \in I_{0}$ and $s \in I_{1} ; \tau_{1}(\underline{1}-$ v) $\geq r, \tau_{1}^{*}(\underline{1}-v) \leq s$ and let $y_{s} \leq \underline{1}-$ $f(v)$. Then $f^{-1}\left(y_{s}\right) \leq \underline{1}-v$, and hence there exists $\mu \in \mathbf{Q}_{\tau_{1}, \tau_{1}^{*}}\left(f^{-1}\left(y_{s}\right), r, s\right)$ such that $f^{-1}\left(y_{s}\right) \leq \mu \leq$ $C_{\tau_{1}, \tau_{1}^{*}}(\mu, r, s) \leq \underline{1}-\nu$. Since $f$ is double fuzzy weakly preclosed by using Theorem 3.12, there exists $(r, s)$-fuzzy pre-Q-neighborhood $\gamma \in I^{Y}$ with $y_{s} \leq \gamma$ and $f^{-1}(\gamma) \leq$ $C_{\tau_{1}, \tau_{1}^{*}}(\mu, r, s)$. Therefore, we obtain $f^{-1}(\gamma) \bar{q} v$ and hence $\gamma \bar{q} f(v)$, this shows that $y_{s} \notin P C_{\tau_{2}, \tau_{2}^{*}}(f(v), r, s)$. Therefore, $f(v)$ is $(r, s)$-fpc and $f$ is double fuzzy preclosed function.

Definition 4.15. A function $f:\left(X, \tau_{1}, \tau_{1}^{*}\right) \rightarrow\left(Y, \tau_{2}, \tau_{2}^{*}\right)$ is said to be double fuzzy contra-open (resp. double fuzzy contra-closed ) if $\tau_{2}(\underline{1}-f(\lambda)) \geq r$ and $\tau_{2}^{*}(\underline{1}-f(\lambda)) \leq s$ (resp. $\tau_{2}(f(\lambda)) \geq r$ and $\left.\tau_{2}^{*}(f(\lambda)) \leq s\right)$ for each $\lambda \in I^{X}, r \in I_{0}$ and $s \in I_{1} ; \tau_{1}(\lambda) \geq r$ and $\tau_{1}^{*}(\lambda) \leq s$ (resp. $\tau_{1}(\underline{1}-\lambda) \geq r$ and $\left.\tau_{1}^{*}(\underline{1}-\lambda) \leq s\right)$.

Theorem 4.19. Iff $:\left(X, \tau_{1}, \tau_{1}^{*}\right) \rightarrow\left(Y, \tau_{2}, \tau_{2}^{*}\right)$ is double fuzzy contra-open, then $f$ is double fuzzy weakly preclosed.

Proof. Let $\lambda \in I^{X}, r \in I_{0}$ and $s \in I_{1}$ such that $\tau_{1}(\underline{1}-\lambda) \geq$ $r$ and $\tau_{1}^{*}(\underline{1}-\lambda) \leq s$. Then, 


$$
\begin{aligned}
P C_{\tau_{2}, \tau_{2}^{*}}\left(f\left(I_{\tau_{1}, \tau_{1}^{*}}(\lambda, r, s)\right), r, s\right) & \leq f\left(I_{\tau_{1}, \tau_{1}^{*}}(\lambda, r, s)\right) \\
& \leq f(\lambda) .
\end{aligned}
$$

Theorem 4.20. If $:\left(X, \tau_{1}, \tau_{1}^{*}\right) \rightarrow\left(Y, \tau_{2}, \tau_{2}^{*}\right)$ is double fuzzy weakly preclosed, then for every $v \in I^{Y}$ and every $\lambda \in I^{X}, r \in I_{0}, s \in I_{1}$ such that $\tau_{1}(\lambda) \geq r$ and $\tau_{1}^{*}(\lambda) \leq s$ with $f^{-1}(\nu) \leq \lambda$, there exists $(r, s)$-fpc set $\gamma \in I^{Y}$ such that $v \leq \gamma$ and $f^{-1}(\gamma) \leq C_{\tau_{1}, \tau_{1}^{*}}(\lambda, r, s)$.

Proof. Let $v \in I^{Y}$ and let $\lambda \in I^{X}, r \in I_{0}$ and $s \in I_{1}$ such that $\tau_{1}(\lambda) \geq r$ and $\tau_{1}^{*}(\lambda) \leq s$ with $f^{-1}(v) \leq$ $\lambda$. Put $\gamma=P C_{\tau_{2}, \tau_{2}^{*}}\left(f\left(I_{\tau_{1}, \tau_{1}^{*}}\left(C_{\tau_{1}, \tau_{1}^{*}}(\lambda, r, s), r, s\right)\right), r, s\right)$, then $\gamma$ is $(r, s)$-fpc set in $I^{Y}$ such that $\nu \leq \gamma$ since $v \leq f(\lambda) \leq f\left(I_{\tau_{1}, \tau_{1}^{*}}\left(C_{\tau_{1}, \tau_{1}^{*}}(\lambda, r, s), r, s\right)\right) \leq$ $P C_{\tau_{2}, \tau_{2}^{*}}\left(f\left(I_{\tau_{1}, \tau_{1}^{*}}\left(C_{\tau_{1}, \tau_{1}^{*}}(\lambda, r, s), r, s\right)\right), r, s\right)=\gamma$. And since $f$ is double fuzzy weakly preclosed, $f^{-1}(\gamma) \leq C_{\tau_{1}, \tau_{1}^{*}}(\lambda, r, s)$.

Corollary 4.21. If $f:\left(X, \tau_{1}, \tau_{1}^{*}\right) \rightarrow\left(Y, \tau_{2}, \tau_{2}^{*}\right)$ is double fuzzy weakly preclosed, then for every $y_{s} \in \mathbf{P}(Y)$ and every $\lambda \in I^{X}, r \in I_{0}$ and $s \in I_{1}$ such that $\tau_{1}(\lambda) \geq r$ and $\tau_{1}^{*}(\lambda) \leq s$ with $f^{-1}\left(y_{s}\right) \leq \lambda$, there exists $(r, s)$-fpc set $\gamma \in I^{Y} ; y_{s} \leq \gamma$ such that $f^{-1}(\gamma) \leq C_{\tau_{1}, \tau_{1}^{*}}(\lambda, r, s)$.

Definition 4.16. A fuzzy set $\lambda \in I^{X}$ is called $(r, s)$ fuzzy $\theta$-compact if for each family $\left\{\mu_{i} \mid i \in J\right\}$ in $\{\mu \in$ $\left.I^{X} \mid \mu \in \mathbf{Q}_{\tau, \tau^{*}}(\lambda, r, s)\right\}$ satisfy $\left(\bigvee_{i \in J} \mu_{i}\right)(x) \geq \lambda(x)$ for each $x \in X$, there exist a finite subset $J_{0}$ of $J$ such that $\lambda \leq I_{\tau, \tau^{*}}\left(\bigvee\left\{C_{\tau, \tau^{*}}\left(\mu_{i}, r, s\right) \mid i \in J_{0}\right\}, r, s\right)$.

Theorem 4.22. Iff $:\left(X, \tau_{1}, \tau_{1}^{*}\right) \rightarrow\left(Y, \tau_{2}, \tau_{2}^{*}\right)$ is double fuzzy weakly preclosed with all fibers $(r, s)$-fuzzy $\theta$-closed, then $f(\lambda)$ is $(r, s)$-fpcfor each $(r, s)$-fuzzy $\theta$-compact $\lambda \in I^{X}$, $r \in I_{0}$ and $s \in I_{1}$.

Proof. Let $\lambda$ be $(r, s)$-fuzzy $\theta$-compact and let $y_{s} \leq \underline{1}-$ $f(\lambda)$. Then $f^{-1}\left(y_{s}\right) \bar{q} \lambda$ and for each $x_{t} \leq \lambda$ there is $\mu_{x_{t}} \in$ $\mathbf{Q}_{\tau_{1}, \tau_{1}^{*}}\left(x_{t}, r, s\right)$ with $x_{t} \leq \mu_{x_{t}}$ and $C_{\tau_{1}, \tau_{1}^{*}}\left(\mu_{x_{t}}, r, s\right) \bar{q} f^{-1}\left(y_{s}\right)$. Clearly $\left\{\mu_{x_{t}} \mid x_{t} \leq \lambda, \mu_{x_{t}} \in \mathbf{Q}_{\tau_{1}, \tau_{1}^{*}}(\lambda, r, s)\right\}$ satisfy $\left(\bigvee_{i \in J} \mu_{i}\right)(x) \geq \lambda(x)$ for each $x \in X$ and since $\lambda$ is $(r, s)$ fuzzy $\theta$-compact, there is $\left\{\mu_{x_{1}}, \mu_{x_{2}}, \mu_{x_{3}}, . ., \mu_{x_{n}}\right\} \subseteq\left\{\mu_{x_{t}} \mid\right.$ $\left.x_{t} \leq \lambda, \mu_{x_{t}} \in \mathbf{Q}_{\tau_{1}, \tau_{1}^{*}}(\lambda, r, s)\right\}$ such that $\lambda \leq I_{\tau_{1}, \tau_{1}^{*}}(\xi, r, s)$, where $\xi=\bigvee\left\{C_{\tau_{1}, \tau_{1}^{*}}\left(\mu_{x_{i}}, r, s\right) \mid i=1,2, \ldots, n\right\}$. Since $f$ is double fuzzy weakly preclosed, by using Theorem 3.12 there exists $\gamma \in \mathbf{P Q}_{\tau_{1}, \tau_{1}^{*}}\left(y_{s}, r, s\right)$ with

$$
\begin{aligned}
f^{-1}\left(y_{s}\right) \leq f^{-1}(\gamma) & \leq C_{\tau_{1}, \tau_{1}^{*}}(\underline{1}-\xi, r, s)=\underline{1}-I_{\tau_{1}, \tau_{1}^{*}}(\xi, r, s) \\
& \leq \underline{1}-\lambda .
\end{aligned}
$$

Therefore $y_{s} \leq \gamma$ and $\gamma \bar{q} f(\lambda)$. Thus $y_{s} \leq 1-$ $P C_{\tau_{2}, \tau_{2}^{*}}(f(\lambda), r, s)$. Thus $f(\lambda)$ is $(r, s)$-fpc set.
Definition 4.17. Let $\left(X, \tau, \tau^{*}\right)$ be an I-dfts. The fuzzy sets $\lambda, \mu \in I^{X}$ are $(r, s)$-fuzzy strongly separated if there exist $v, \gamma \in I^{X}$ such that $\tau(v) \geq r$ and $\tau^{*}(v) \leq s, \tau(\gamma) \geq r$ with $\lambda \leq \nu, \mu \leq \gamma$ and $C_{\tau, \tau^{*}}(\nu, r, s) \bar{q} C_{\tau, \tau^{*}}(\gamma, r, s)$.

Definition 4.18. An I-dfts $\left(X, \tau, \tau^{*}\right)$ is called $(r, s)$-fuzzy pre $T_{2}$ iffor each $x_{t_{1}}, x_{t_{2}}$ with different supports there exists $(r, s)$-fpo sets $\lambda, \mu \in I^{X}$ such that $x_{t_{1}} \leq \lambda \leq x_{1-t_{2}}, x_{t_{2}} \leq$ $\mu \leq x_{1-t_{1}}$ and $\lambda \bar{q} \mu$.

Theorem 4.23. If $:\left(X, \tau_{1}, \tau_{1}^{*}\right) \rightarrow\left(Y, \tau_{2}, \tau_{2}^{*}\right)$ is double fuzzy weakly preclosed surjection and all fibers are $(r, s)$ fuzzy strongly separated, then $\left(Y, \tau_{1}, \tau_{1}^{*}\right)$ is $(r, s)$-fuzzy pre$T_{2}$.

Proof. Let $y_{s_{1}}, y_{s_{2}} \in \mathbf{P}(Y)$ and let $\gamma, \nu \in I^{X}, r \in$ $I_{0}$ and $s \in I_{1} ; \tau_{1}(\gamma) \geq r, \tau_{1}^{*}(\gamma) \leq s, \tau_{1}(v) \geq$ $r$ and $\tau_{1}(\nu) \leq s$ such that $f^{-1}\left(y_{s_{1}}\right) \leq \gamma$ and $f^{-1}\left(y_{s_{2}}\right) \leq v$ respectively with $C_{\tau_{1}, \tau_{1}^{*}}(\gamma, r, s) \bar{q} C_{\tau_{1}, \tau_{1}^{*}}(v, r, s)$. By using Theorem 3.12-4 there are $(r, s)$-fpo sets $\lambda, \mu \in$ $I^{Y}$ such that $y_{s_{1}} \leq \lambda$ and $y_{s_{2}} \leq \mu, f^{-1}(\lambda) \leq$ $C_{\tau_{1}, \tau_{1}^{*}}(\gamma, r, s)$ and $f^{-1}(\mu) \leq C_{\tau_{1}, \tau_{1}^{*}}(\nu, r, s)$. Therefore $\lambda \bar{q} \mu$, because $C_{\tau_{1}, \tau_{1}^{*}}(\gamma, r, s) \bar{q} C_{\tau_{2}, \tau_{2}^{*}}(\nu, r, s)$ and $f$ is surjective. Thus $\left(Y, \tau_{2}, \tau_{2}^{*}\right)$ is $(r, s)$-fuzzy pre- $T_{2}$.

Definition 4.19. an I-dfts $\left(X, \tau, \tau^{*}\right)$ is said to be $(r, s)$ extremally disconnected if $\tau\left(C_{\tau, \tau^{*}}(\lambda, r, s)\right) \geq r$ and $\tau^{*}\left(C_{\tau, \tau^{*}}(\lambda, r, s)\right) \leq s$ for each $\lambda \in I^{X} ; \tau(\lambda) \geq r$ and $\tau^{*}(\lambda) \leq s$.

Definition 4.20. an I-dfts $\left(X, \tau, \tau^{*}\right)$ is said to be $(r, s)$ fuzzy almost compact if for each $(r, s)$-fuzzy open cover $\left\{\lambda_{i} \mid i \in J\right\}$ of $X$, there is a finite subset $J_{0}$ of $J$ such that $\bigvee\left\{C_{\tau, \tau^{*}}\left(\lambda_{i}, r, s\right) \mid i \in J_{0}\right\}=\underline{1}$.

Definition 4.21. A fuzzy set $\lambda$ in an I-dfts $\left(X, \tau, \tau^{*}\right)$ is said to be $(r, s)$-fuzzy p-compact iff for each family of $(r, s)$ fpo sets $\left\{\mu_{i} \mid i \in J\right\}$ satisfies $\left(\bigvee_{i \in J} \mu_{i}\right)(x)=\lambda(x)$ for each $x \in X$. There exists finite subfamily $J_{0}$ of $J$ such that $\left(\bigvee_{i \in J_{0}} P C_{\tau, \tau^{*}}\left(\mu_{i}, r, s\right)\right)(x) \geq \lambda(x)$ for each $x \in X$.

Theorem 4.24. Let $\left(X, \tau_{1}, \tau_{1}^{*}\right)$ be $(r, s)$-extremally disconnected I-dfts. Letf $:\left(X, \tau_{1}, \tau_{1}^{*}\right) \rightarrow\left(Y, \tau_{2}, \tau_{2}^{*}\right)$ be double fuzzy open and double fuzzy preclosed injective function such that $f^{-1}\left(y_{s}\right)$ is $(r, s)$-fuzzy almost compact for each $y_{s} \in \mathbf{P}(Y)$. If $\lambda \in I^{Y}$ is $(r, s)$-fuzzy P-compact. Then $f^{-1}(\lambda)$ is $(r, s)$-fuzzy almost compact.

Proof. Let $\left\{v_{j} \mid æ \in J\right\}$ be $(r, s)$-fuzzy open cover of $f^{-1}(\lambda)$. Then for each $y_{s} \leq \lambda \wedge f(X), f^{-1}\left(y_{s}\right) \leq$ $\bigvee\left\{C_{\tau_{1}, \tau_{1}^{*}}\left(v_{j}, r, s\right) \quad \mid\right.$ æe $\left.\in J\left(y_{s}\right)\right\}=\gamma_{y_{s}}$, for some finite subfamily $J\left(y_{s}\right)$ of $J$. Since $\left(X, \tau_{1}, \tau_{1}^{*}\right)$ is $(r, s)$ extremally disconnected each $\tau_{1}\left(C_{\tau_{1}, \tau_{1}^{*}}\left(v_{j}, r, s\right)\right) \geq r$ and 
$\tau_{1}^{*}\left(C_{\tau_{1}, \tau_{1}^{*}}\left(v_{j}, r, s\right)\right) \leq s$, hence $\tau_{1}\left(\gamma_{y_{s}}\right) \geq r$ and $\tau_{1}^{*}\left(\gamma_{y_{s}}\right) \leq s$. So by Corollary 4.21 there exists $(r, s)$-fpc set $\mu_{y_{s}} ; y_{s} \leq \mu_{y_{s}}$ such that $f^{-1}\left(\mu_{y_{s}}\right) \leq C_{\tau_{1}, \tau_{1}^{*}}\left(\gamma_{y_{s}}, r, s\right)$. Then, $\left\{\mu_{y_{s}} \mid y_{s} \leq \lambda \wedge\right.$ $f(X)\} \vee\{\underline{1}-f(X)\}$ is $(r, s)$-fuzzy preclosed cover of $\lambda, \lambda \leq$ $\bigvee\left\{C_{\tau_{2}, \tau_{1}}\left(\mu_{y_{s}}, r, s\right) \mid y_{s} \leq \lambda \wedge f(X)\right\} \vee\left\{C_{\tau_{2}, \tau_{2}^{*}}(\underline{1}-f(X), r, s)\right\}$ for some finite fuzzy subset $K$ of $\lambda \wedge f(X)$. Hence,

$$
\begin{aligned}
f^{-1}(\lambda) & \leq \bigvee_{y_{s} \in K^{-1}} f^{-1}\left(C_{\tau_{2}, \tau_{2}^{*}}\left(\mu_{y_{s}}, r, s\right)\right) \\
& \vee\left\{f^{-1}\left(C_{\tau_{2}, \tau_{2}^{*}}(\underline{1}-f(X), r, s)\right)\right\} \\
& \leq \bigvee_{y_{s} \in K} C_{\tau_{1}, \tau_{1}^{*}}\left(f^{-1}\left(\mu_{y_{s}}\right), r, s\right) \\
& \vee\left\{C_{\tau_{1}, \tau_{1}^{*}}\left(f^{-1}(\underline{1}-f(X)), r, s\right)\right\} \\
& \leq \bigvee_{y_{s} \in K} C_{\tau_{1}, \tau_{1}^{*}}\left(f^{-1}\left(\mu_{y_{s}}\right), r, s\right)
\end{aligned}
$$

so $f^{-1}(\lambda) \leq \bigvee_{æ \in J\left(y_{s}\right), y_{s} \in K} C_{\tau_{1}, \tau_{1}^{*}}\left(v_{æ}, r, s\right)$. Therefore $f^{-1}(\lambda)$ is $(r, s)$-fuzzy almost compact.

\section{Competing interests}

The authors declare that they have no competing interests.

\section{Acknowledgements}

The author would like to thank the reviewers for their valuable comments and helpful suggestions for improvement of the original manuscript.

Received: 23 May 2012 Accepted: 20 July 2012

Published: 25 September 2012

\section{References}

Bhaumik RN, Abbas SE (2008) ( $L, M$ )-intuitionistic fuzzy topological spaces. Bull Kerala Mathematics Assoc 5: 25-47

Chang CL (1968) Fuzzy topological spaces. J Math Anal Appl 24: 39-90

Çoker D, Demirci M (1996) An introduction to intuitionistic fuzzy topological spaces in Šostak's sense. Busefal 67: 67-76

Du S, Qin Q, Wang Q, Li B (2005) Fuzzy description of topological relations i: a unified fuzzy 9-intersection model. Lecture Notes Comp Sci 3612: 1261-1273

Egenhofer MJ, Franzosa R (1991) Point-set topological spatial relations. J Geo Info Sys 2: 161-174

El-Naschie MS (1998) On the uncertainty of cantorian geometry and the twoslit experiment. Chaos, Solitons Fractals 9: 517-529

El-Naschie, MS (2000) On the certification of heterotic strings, M theory and $e^{\infty}$ theory. Chaos, Solitons and Fractals: 2397-2408

Garcia JG, Rodabaugh SE (2005) Order-theoretic, topological, categorical redundancies of interval-valued sets, grey sets, vague sets, interval-valued "intuitionistic" sets, "intuitionistic" fuzzy sets and topologies. Fuzzy Sets Syst 156: 445-484

Herring J, Egenhofer MJ (1991) Categorizing binary topological relations between regions, lines and points in geographic databases. Tech Report, Dept. of Surveying Engineering, Univ. of Maine, Orono, ME

Kubiak T (1985) On fuzzy topologies. Ph.D thesis, A. Mickiewicz, poznan

Lee EP, Im YB (2001) Mated fuzzy topological spaces. Int J Fuzzy Logic Intell Syst 11: 161-165

Lowen R (1976) Fuzzy topological spaces and fuzzy compactness. J Math Anal Appl 56: 621-623

Samanta SK, Mondal TK (1997) Intuitionistic gradation of openness: intuitionistic fuzzy topology. Busefal 73: 8-17

Samanta, SK, Mondal, TK (2002) On intuitionistic gradation of openness. Fuzzy Sets Syst 131: 323-336
Šostak AP (1985) On a fuzzy topological structure. Suppl Rend Circ Matem Palermo-Sir II 11: 89-103

Tang X (2004) Spatial object modeling in fuzzy topological spaces with applications to land cover change in China. Ph.d. dissertation, ITC Dissertation No. 108, Univ. of Twente, The Netherlands

doi:10.1186/2193-1801-1-19

Cite this article as: Ghareeb: Weak forms of continuity in I-double gradation fuzzy topological spaces. SpringerPlus 2012 1:19.

\section{Submit your manuscript to a SpringerOpen ${ }^{\circ}$ journal and benefit from:}

- Convenient online submission

- Rigorous peer review

- Immediate publication on acceptance

- Open access: articles freely available online

- High visibility within the field

- Retaining the copyright to your article

Submit your next manuscript at $\boldsymbol{\nabla}$ springeropen.com 